\title{
Effect of Korteweg stress on viscous fingering of solute plugs in a porous medium
}

\author{
S. Swernath ${ }^{\mathrm{a}}$, B. Malengier ${ }^{\mathrm{b}}$, S. Pushpavanam ${ }^{\mathrm{a}}$ \\ ${ }^{a}$ Department of Chemical Engineering, Indian Institute of Technology Madras, Chennai \\ 600036 \\ ${ }^{b}$ Department of Mathematical Analysis, Research Group NfaM ${ }^{2}$, Ghent University, \\ Galglaan 2, B-9000 Ghent, Belgium
}

\begin{abstract}
In this work we first present a unified framework for the analysis of miscible and immiscible viscous fingering. To establish this we introduce the Korteweg stresses for miscible fluids which arise due to concentration gradients and are analogous to surface tension between two immiscible fluids. For the immiscible fingering we use the continuum representation of surface tension which treats surface tension as a body force. This analogy between the two types of fingering helps us obtain a better insight into the role of Korteweg stresses in miscible fluids and enables in resolving ambiguities in the role of these stresses in a dynamic system. Finally we examine the effect of Korteweg stresses to see how they can be a stabilizing influence for miscible viscous fingering of finite slices in a chromatography column and discuss if this can give an extra control option to improve the performance of the separation.
\end{abstract}

Key words: Continuum theory of surface tension, Korteweg stress, Viscous fingering, Chromatographic columns

\section{Introduction}

Viscous fingering has been extensively investigated in the past in the context of both miscible and immiscible fluids. In the former the behavior is described by the Peclet number Pe which is a ratio of convective to diffusive

Email addresses: bm@cage.UGent.be (B. Malengier), spush@iitm.ac.in (S. Pushpavanam)

$U R L: \mathrm{http}: / /$ cage.ugent.be/ bm/ (B. Malengier) 
fluxes. Here the high values of diffusion coefficients can reduce concentration gradients and mitigate the effects of viscous fingering. Immiscible fingering on the other hand is determined by the Capillary number $\mathrm{Ca}$ which is a ratio of viscous forces to surface tension forces (Nagatsu et al. (2008)). Most of the work done so far has progressed with the understanding that the two phenomena are qualitatively similar. The physical processes governing the flow are different and the mathematical formulations that have been used so for describing the flows are distinct. Thus for miscible fingering we solve a single set of momentum and continuity equations along with a species balance. Here the viscosity dependence on concentration gives rise to irrotational effects as a result of which a vorticity is generated. For immiscible fingering the usual approach involves solving two sets of equations, one for each fluid incorporating a boundary condition which takes into account the effect of surface tension (Yih (1980); Homsy (1987)).

Miscible viscous fingering occurs when a low viscosity fluid invades a high viscosity fluid. The difference in viscosity is generated by the concentration of a solute in the fluid. The solute concentration contours appear in the form of fingers. In a porous medium or a Hele-Shaw cell under these conditions the fluid does not exhibit a plug flow (uniform) velocity profile. Transverse velocity components are generated as a result of the fingering process. Extensive studies have been carried out experimentally in Shalliker et al. (2007a,b) as well as theoretically in Tan and Homsy (1988); Zimmerman and Homsy (1991) to understand the behavior of the system.

The effect of Korteweg stresses has been recently analyzed in the context of miscible fingering. These stresses act as a body force and mimic a surface tension like behavior. They are generated by concentration gradients in the fluid. Chen et al. (2001) have found that Korteweg stresses can stabilize fingering of a miscible drop in a porous medium. The role of Korteweg stresses in the presence of density changes has been discussed in detail by Joseph et al. (1996). In their formulation they emphasize that the velocity field may not be solenoidal for a mixture of incompressible fluids whose densities are not equal. Here the Korteweg stress manifests itself as a gradient of a pressure like term as well as a stress tensor. The effect of these stresses are dominant in a microgravity environment (Bessonov et al. (2005)).

Brackbill et al. (1992) have proposed a continuum theory of surface tension which allows us to view interfacial tension as a body force in the region where an interface is present. This approach is also the basis of the numerical scheme of Volume Of Fluid approach used in solving multi phase 
flow problems (Guan and Pitchumani (2003)). In a similar context it was shown that multiphase flow problems can be simulated by introducing an additional stress tensor in the momentum equation by Nadiga and Zaleski (1996) using thermodynamic considerations. Zou and He (1999) used kinetic considerations to formally obtain these additional stress terms.

There have been a significant number of experimental studies focussing on viscous fingering in chromatographic columns. Imaging techniques have enabled studies of viscous fingering by visualizing the concentration or temperature profiles within the column (Shalliker et al. (2007a,b)). The linear stability analysis performed by Tan and Homsy (1986) was applied to chromatographic column to predict the onset of instability by Rousseaux et al. (2007). The effect of adsorption of a solute in a porous medium for a linear isotherm has also been recently analyzed, Mishra et al. (2007). To simulate a solute plug moving in a chromatography column a non-linear analysis was performed with a miscible slice of a fluid with a viscosity different from its environment in Mishra et al. (2008). This could exhibit both forward and backward fingering depending on the value of the slug viscosity relative to its environment. A high accuracy numerical simulation of a fluid slice of finite width in a porous media was performed to analyze the evolution of concentration by Chen and Wang (2001). All the above studies consider the system to be isothermal.

In this work we first describe a unified framework and formulation which is based on Korteweg stresses for miscible fingering and the continuum theory of surface tension for immiscible fingering. This helps us understand the analogy between the two cases in a precise and clear manner. We then discuss the effect of Korteweg stresses on the effect of viscous fingering in a chromatography column when a plug of a finite length moves along the column surrounded by a buffer with a different viscosity. We analyze these effects quantitatively and summarize the results in a final section containing our conclusions.

\section{Model Formulation}

In the continuum surface tension formulation (Brackbill et al. (1992)) the equations which govern an immiscible system of two liquids are, the equation of continuity

$$
\frac{\partial \rho}{\partial t}+\nabla \cdot(\rho \mathbf{u})=0
$$


and the equation of momentum given by

$$
\frac{\partial \rho \mathbf{u}}{\partial t}+\nabla \cdot(\rho \mathbf{u u})=-\nabla P+\nabla \cdot \pi^{v}+\nabla \cdot \pi^{1},
$$

where the stress tensor $\pi^{1}$ is defined as

$$
\pi^{1}=\lambda\left[\left(\frac{1}{2}|\nabla \rho|^{2}+\rho \nabla^{2} \rho\right) I-\nabla \rho \nabla \rho\right] .
$$

Here, $\lambda$ is a parameter which is proportional to the surface tension between two fluids. Zou and He (1999) have shown that the above set of equations can be obtained from kinetic theory. In these equations $\pi^{v}$ represents the stress tensor generated by viscosity which occurs in the classical Navier stokes equation. The additional stress tensor $\pi^{1}$ arises from the continuum representation of surface tension by considering the density to be diffuse in the neighborhood of the interface. The contribution of this as a body force term can be written as

$$
\nabla \cdot \pi^{1}=\lambda \nabla \cdot\left[\left(\frac{1}{2}|\nabla \rho|^{2}+\rho \nabla^{2} \rho\right) I-\nabla \rho \nabla \rho\right]=\lambda \rho \nabla \nabla^{2} \rho
$$

The term with the identity matrix in the above equation can be expressed as a gradient of $Q_{i}=\left(\frac{1}{2}|\nabla \rho|^{2}+\rho \nabla^{2} \rho\right) \lambda$. Using this we write the above equation as

$$
\nabla \cdot \pi^{(1)}=\nabla Q_{i}-\lambda \nabla \cdot[\nabla \rho \nabla \rho]
$$

The surface tension in this formulation is given by

$$
\lim _{A \rightarrow 0} \frac{1}{A} \int_{\Omega} \lambda \rho \nabla \nabla^{2} \rho=\sigma \kappa_{c} \mathbf{n}
$$

In this formulation $\sigma$ is the surface tension, $\kappa_{c}$ is the curvature of the interface and $\mathbf{n}$ is the outward normal at the interface, $\Omega$ is a thin volume element containing a small part of a curved interface of area $\mathrm{A}$.

In addition to these equations we need an equation which describes how the volume fraction $F$ occupied by a fluid changes with position and time. This is given by

$$
\frac{\partial F}{\partial t}+\nabla \cdot(F \mathbf{u})=0
$$


The density and viscosity in the above formulations are given by $\rho=\rho_{1} * F+$ $\rho_{2} *(1-F), \mu=\mu_{1} * F+\mu_{2} *(1-F)$. The gradients in density and viscosity enter through gradients in $F$. We emphasize that the two fluids cannot diffuse into each other and hence there is no diffusion term in equation (7).

Recently the theory of Korteweg stresses has been applied to the case of two miscible incompressible fluids with varying densities flowing through a porous medium by Joseph et al. (1996). They show that when the densities of the two fluids are unequal the assumption of the velocity being solenoidal may be incorrect. In our work we assume the densities of the two fluids which are miscible to be equal as we are concerned only with viscous fingering. Consequently our velocity field is solenoidal. We can alternatively view this as neglecting expansion effects in our system. We are interested in analyzing the effect of surface tension like Korteweg stresses and hence retain this in our formulation. Here the equation of continuity is given as before, (1). We follow Joseph et al. (1996) in writing the equation of momentum as

$$
\frac{\partial \rho \mathbf{u}}{\partial t}+\nabla \cdot(\rho \mathbf{u u})=-\nabla P+2 \nabla \cdot(\mu D[\mathbf{u}])+\nabla \cdot T^{(2)}
$$

Here $D[\mathbf{u}]$ represents the symmetric part of the stress tensor and the term associated with it is equivalent to $\pi^{v}$ in (2). Following Joseph et al. (1996) the additional stress tensor $T^{(2)}$ is defined as

$$
T^{(2)}=\left[-\frac{1}{3} \hat{\delta}|\nabla \phi|^{2}+\frac{2}{3} \gamma \nabla^{2} \phi\right] I+\hat{\delta} \nabla \phi \nabla \phi .
$$

Here $\phi$ represents the composition of the miscible fluid, $\hat{\delta}$ and $\gamma$ are constants when the density of the two fluids making up the mixture are the same. Following the approach for immiscible fluids we again write the body force arising out of the stress tensor as

$$
\nabla \cdot T^{(2)}=\nabla Q_{m}-\hat{\delta} \nabla \cdot[\nabla \phi \nabla \phi],
$$

where $Q_{m}$ represents the terms multiplying the identity matrix in (9). Here $\phi$ is governed by the mass balance equation

$$
\frac{\partial \phi}{\partial t}+\mathbf{u} \cdot \nabla \phi=D \nabla^{2} \phi
$$

Comparing equations (2)-(7) for the immiscible case with (8)-(11) for the miscible case, we see the one to one correspondence between the formal derivation of surface tension as a body force and the phenomenological Korteweg 


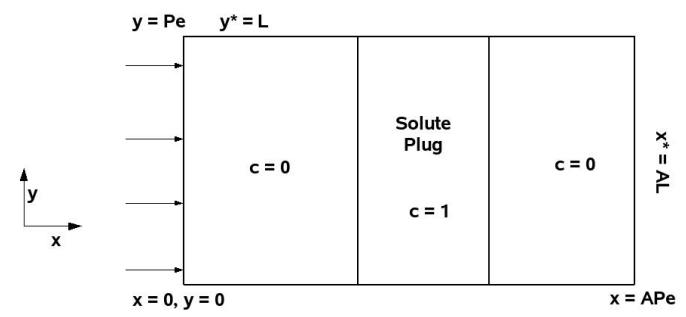

Figure 1: Schematic of the system under study

stresses. The similarity in the formulation of Korteweg stress with continuum theory of surface tension allows us to infer that the parameters $\gamma$ is positive and $\hat{\delta}$ is negative is the only possibility which is admissible from thermodynamic as well as kinetic considerations. The same conclusion was arrived at by $\mathrm{Hu}$ and Joseph (1992) on the basis of the need to avoid Hadamard instability or the mathematical ill-posedness of the problem. It must be pointed out here that $\gamma$ must have the same units as $\phi \hat{\delta}$ to ensure dimensional consistency. If it is exactly equal to $\phi \hat{\delta}$ the analogy with surface tension will be exact and $\hat{\delta}$ will then be analogous to $\lambda$ for the immiscible case.

The interface for the immiscible fingering is tracked by following the evolution of the scalar variable $F$ by purely advection with no diffusion. This is understandable since in a mixture of two immiscible liquids the two liquids do not diffuse into each other. As opposed to this diffusion is an important process governing mass transfer and hence this has to be included in how $\phi$ changes spatially and temporally. Thus we see that in the limit of the diffusion coefficient tending to zero the miscible fingering formulation collapses to a type of immiscible fingering formulation.

\subsection{Application to Chromatography}

We now focus on understanding theoretically the behavior of a chromatographic column in the presence of Korteweg stresses. We consider a homogeneous 2-D porous medium of width $H$ (along $x$ ), length $L$ (along $y$ ) with a constant permeability $\kappa$ (Figure 1$)$. The liquid within the column is considered to be incompressible and flowing from the left with a uniform velocity $U$. The system has two different liquids with distinct properties. These properties are governed by the concentration of a solute. The solute plug in the middle of the column has different viscosity as compared to the buffer solution in which it is present. The concentration of the solute which determines the viscosity is assumed to be zero in the buffer. The evolution 
of the system is governed by the law of conservation of mass and momentum in addition to a species balance. The corresponding equations in a reference frame moving with a constant velocity $U$ in the $x$-direction can then be written as

$$
\begin{gathered}
\nabla \cdot \mathbf{u}=0 \\
\nabla[P+Q(c)]=-\frac{\mu}{\kappa}[\mathbf{u}+\hat{i}]+\nabla \cdot[\hat{\delta}(\nabla c)(\nabla c)] .
\end{gathered}
$$

Here $\hat{i}$ arises due to analysing the system in a moving reference frame, it represents the unit vector in the $x$-direction, and $Q(c)$ is the part of the Korteweg stress which can be written as the gradient of a scalar and hence can be viewed as a pressure term. This term can be defined as

$$
Q(c)=\frac{\hat{\delta}}{3}\left[\left(\frac{\partial c}{\partial x}\right)^{2}+\left(\frac{\partial c}{\partial y}\right)^{2}\right]-\frac{2 \gamma}{3}\left[\frac{\partial^{2} c}{\partial x^{2}}+\frac{\partial^{2} c}{\partial y^{2}}\right]
$$

The evolution of concentration of the solute is given by the advection-diffusion equation

$$
\frac{\partial c}{\partial t}+\mathbf{u} \cdot \nabla c=\nabla^{2} c
$$

The variation of viscosity is assumed to be of the form $\mu=\mu_{0} e^{-R c}$. These equations are converted to the stream-function vorticity formulation using the general convention for $\psi$ and $\omega$. Since the Korteweg stress term $Q(c)$, containing $\gamma$, occurs as a gradient term this drops out of the calculations and hence does not affect the results. The resulting dimensionless equations used for the non-linear simulation can be described as

$$
\nabla^{2} \psi=-\omega
$$

$$
\begin{gathered}
\omega=-R\left[\nabla \psi \cdot \nabla c+\frac{\partial c}{\partial y}\right] \\
-\frac{\delta}{\mu}\left[\frac{\partial c}{\partial x}\left(\frac{\partial^{3} c}{\partial x^{2} \partial y}+\frac{\partial^{3} c}{\partial y^{3}}\right)-\frac{\partial c}{\partial y}\left(\frac{\partial^{3} c}{\partial x \partial y^{2}}+\frac{\partial^{3} c}{\partial x^{3}}\right)\right] \\
\frac{\partial c}{\partial t}+\frac{\partial \psi}{\partial y} \frac{\partial c}{\partial x}-\frac{\partial \psi}{\partial x} \frac{\partial c}{\partial y}=\nabla^{2} c
\end{gathered}
$$

The non-dimensionalization has been carried out using $\frac{D}{U}$ as the length scale and $\frac{D}{U^{2}}$ as the time scale as there are no geometrically occurring length 
scales which define the physical process. $P e$ is defined as $P e=\frac{U L}{D}$. The parameter $P e$ is the dimensionless width of the domain and $A P e$ determines the dimensionless length of the domain, $A=\frac{H}{L}$, where $H(L)$ represents the dimensional length (width) of the domain. Consequently in our formulation the $P e$ appears in the boundary condition. In the term representative of the Korteweg stress the dimensionless parameter $\delta$ is defined as $\delta=\frac{\kappa U^{2}}{\mu_{0} D^{3}} \hat{\delta}$. This is different from the dimensionless parameter $\left(\delta_{\text {chen }}\right)$ used in Chen et al. (2001) who used the drop diameter as the characteristic length scale. Our parameter $\delta$ can be viewed as $\delta=\delta_{\text {chen }} \times(P e)^{3}$ where $P e$ is defined as $P e=\frac{U L}{D}$. Since $P e$ is $\mathrm{O}\left(10^{3}\right)$ and $\delta_{\text {chen }}$ of Chen et al. (2001) is $\mathrm{O}\left(10^{-4}\right)$ we see that our $\delta$ is $\mathrm{O}\left(10^{5}\right)$. This will be the magnitude of the $\delta$ we will use to be consistent with what has been reported in the earlier studies involving Korteweg stresses.

\subsection{Initial and Boundary conditions}

The concentration in the zones occupied by the slug in the middle is initialized to 1 and the remaining zones are initialized to 0 . Throughout our simulations we have used the middle one-third of the system to be occupied by the solute slug. As we use the moving reference frame co-ordinates the initial flow within the column is zero. Hence $\psi=0$ is taken throughout the column as the initial condition for $\psi$. A set of random numbers is generated at the interface separating the slug and the remaining fluid. The random number is assumed at both the ends of the slug. This creates the necessary disturbance to study the non-linear evolution of the spatial patterns.

We use no-flux boundary conditions for the concentration along the $y$ direction ( $y=0$ and $y=P e$, the direction transverse to the flow). $\psi$ values are taken to be zero at these boundaries implying no flow across the domain in the axial direction in the moving reference frame. Along the $x$-direction (axial) direction we impose Dirichlet boundary condition. The concentration as well as stream function are assumed to be zero at both the ends along the axial direction $(c=0$ and $\psi=0$ at $x=0$ and $x=A P e)$.

\subsection{Non-linear simulations}

The governing equations (16)-(18) are solved numerically using a finite difference scheme. The typical finite difference scheme to calculate the mixed 
third order derivatives arising in the equations is given by

$$
\begin{aligned}
& \left.\frac{\partial^{3} c}{\partial x \partial y^{2}}\right|_{i, j}=\frac{\partial}{\partial x}\left[\frac{\partial^{2} c}{\partial y^{2}}\right]_{i, j}=\frac{\partial}{\partial x}\left[\frac{c_{\bullet, j+1}-2 c_{\bullet, j}+c_{\bullet, j-1}}{\Delta y^{2}}\right]_{i} \\
& =\left[\frac{c_{i+1, j+1}-c_{i-1, j+1}}{2 \Delta x \Delta y^{2}}\right]-2\left[\frac{c_{i+1, j}-c_{i-1, j}}{2 \Delta x \Delta y^{2}}\right]+\left[\frac{c_{i+1, j-1}-c_{i-1, j-1}}{2 \Delta x \Delta y^{2}}\right]
\end{aligned}
$$

and likewise for the other terms. Here the index $i(j)$ is used to represent the grid number in the $x(y)$ direction. Similarly $\Delta x$ and $\Delta y$ are the spacing in the $x$ and $y$ directions. The time variations are updated through a first order forward difference scheme. A total number of 256 grids is used along the axial direction ( $x$-direction). Along the transverse direction ( $y$-direction) 128 grids have been used. The algorithm employed is the same as the one detailed in Swernath and Pushpavanam (2007).

\section{Results}

We now discuss the results of the simulations that we have performed for the chromatography column. The slug length is always maintained at $33 \%$ of the total length of the domain located symmetrically about the center. The aspect ratio is fixed for all the simulations at $A=2$. At the outset we would like to determine the role of Korteweg stress in the absence of any viscosity gradients. The viscosity of the slug and that of the fluid are assumed to be the same and hence here $R=0$. We have chosen $P e=1000$ for these simulations. Figure 2(a) depicts the transversely averaged concentration profiles of the solute at a time instant $t=500$. Here we have taken the average of the concentrations prevailing at the time instant in the y direction and depict this as a function of the axial position. For the case when $\delta=0$ the spreading of the solute occurs purely by diffusion in the moving reference frame. From Eq. 17 it appears that the Korteweg stress acts as a physical source of irrotationality and can mathematically induce vorticity or irrotationality. However our results on the averaged concentration profiles for the case when $\delta=-10,000$ [Fig. 2(a)] shows that the spreading of the concentration is purely by diffusion and there is no irrotationality induced in the flow field. Under these conditions when no viscosity gradients are present we observe that the Korteweg stress does not significantly alter the system behavior. We observe that the two concentration profiles overlap exactly over one another implying that the transversely averaged concentration is 


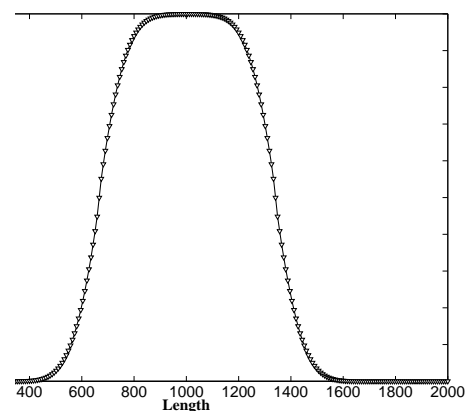

(a)

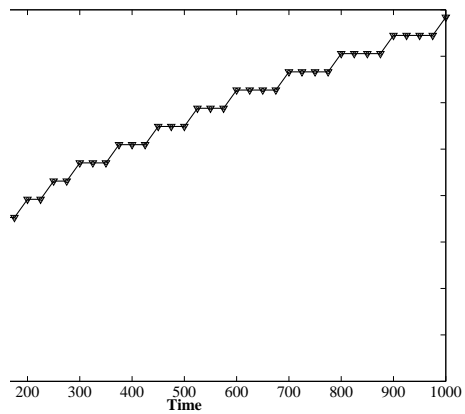

(b)

Figure 2: Pure diffusive flow with and without Korteweg stress with the parameters fixed at $R=0, P e=1000$,

(a) Band broadening in the presence and absence of Korteweg stress at $t=500$. The line depicts the concentration profile for $\delta=0$ and the symbol depicts the profile for $\delta=-10000$.

(b) Mixing length for two interfaces. Both interfaces overlap with each other. The line depicts the interface (both front and back) when $\delta=0$ and the symbol depicts the interfaces for $\delta=-10000$ 
governed purely by the diffusive interfacial movement. This is perhaps not surprising as Korteweg stresses are physically similar to surface tension and hence have a stabilizing influence on the flow and cannot induce irrotationality or fingers in the flow field. We see that the two interfaces (i.e., the front and rear portions of the slug) are spreading out at the same rate as they are symmetric. To confirm this spreading by diffusion we have calculated the mixing length of the system at different instants of time. This is depicted in Fig. 2(b). We follow Mishra et al. (2008) to determine the mixing length of the system. The forward mixing length is defined as the distance between the initial position of the front interface and the farthest position at the right where the transversely average concentration is $c=0.01$. Similarly the backward mixing length is defined as the interval between the initial position of the rear interface and the farthest position on the left where the transverse average concentration is $c=0.01$. While the mixing length is calculated at every time instant of the simulation the plot depicts the value in dimensionless time intervals of 25 units. Here we see that the mixing length varies as $t^{1 / 2}$ confirming that mixing is occurring purely by diffusion. From Fig. 2(b) we again conclude that the inclusion of Korteweg stress does not influence the flow field as the mixing length is not altered by it. Here we would like to emphasize that the dimensionless parameter $\delta$ is defined as $\delta=\frac{\kappa U^{2}}{\mu_{0} D^{3}} \hat{\delta}$ and as explained before is different from what has been used in the literature earlier. Hence although the values of the parameter used in the simulations appear to be high we are consistent with the values reported in the literature.

We now turn our attention to the case when $R \neq 0$. The front face is the unstable interface for $R>0$ (as here the low viscous fluid is pushing the high viscous fluid) and is the stable interface for $R<0$. Similarly the rear face is stable for $R>0$ and unstable for $R<0$. We focus on the situation when $R>0$. Now we have a less viscous plug pushing the more viscous buffer in the porous bed. As a result the interface on the right exhibits fingering instability and we call this forward fingering. Figure 3 depicts the effect of Korteweg stress on the flow behavior when we have have forward fingering. For $R=2$, $A=2$ we depict the behavior of the system for two different values $\delta=0$ and $\delta=-10000$. Transverse averaged concentration profiles have been depicted at $t=750$ for $P e=1000$. Transversely averaged concentration profiles helps us understand the practical problem of band broadening which characterize spreading in chromatographic columns. Mishra et al. (2008) have used the existence of the constant concentration plateau to determine if the two fronts 
(at the front and rear faces) start interacting with each other. It can be seen from Fig. 3(a) that the flat plateau exists for the fingering in the presence of Korteweg stress whereas the flat concentration plateau ceases to exist in the absence of the Korteweg stress. Mixing length defined earlier can be taken to be a measure of the length of the fingering zone. In Fig. 3(b) we see that the rear interface is stable and this grows as $t^{1 / 2}$ and is purely governed by diffusion. For the front interface which undergoes fingering the difference in the band broadening profiles manifests in the difference in the mixing length dependency on time. The mixing length increases faster than the regular diffusion process due to viscous fingering. It can also be seen that the mixing length of the front face at every instant of time is lowered with the inclusion of the extra Korteweg stress term. This basically implies that the fingering is reduced due to the incorporation of the Korteweg stress. Thus the existence of the concentration plateau in Fig. 3(a) and the lower mixing length in Fig. 3(b) both depict the stabilizing influence of Korteweg stress. This behavior has been observed over a wide range of values of $P e$.

Figure 4 depicts an alternate representation of the fingering phenomena. Here the concentration contours in the range 0.1-0.3 are depicted for $\mathrm{Pe}=$ 500, $R=2, A=2$, at $t=200$. These contours represent the locus of points on which the concentration is a constant. The concentration profile within the column is dependent on whether Korteweg stresses are active or not. The constant concentration contours are representative of an interface and we see that in the absence of Korteweg stresses these show a finger like structure. In Fig. 4(a) the fingering of the front face is clearly present when $\delta=0$. In Fig. 4(b) we depict the contours when the Korteweg stresses are introduced. We see that these stresses have a stabilizing influence and the interface is now less deformed. The stabilization of the interface observed here is consistent with the existence of the plateau and the reduction in the mixing length observed earlier.

In addition to the concentration contours we can also analyze the system by viewing the streamlines or the stream functions. The constant streamline contours are depicted in Figure 5. The gradients of the stream function represent the magnitude of velocity. We see that when the Korteweg stresses are absent the gradients of the stream functions are larger and the values of the stream function vary from -9 to +9 in Fig. 5(a) while when the Korteweg stresses are present they vary from -1 to +1 as in Fig. 5(b). This again confirms the stabilizing influence of the Korteweg stresses on the system behavior. The larger values of $\psi$ when $\delta=0$ signifies a higher value of 


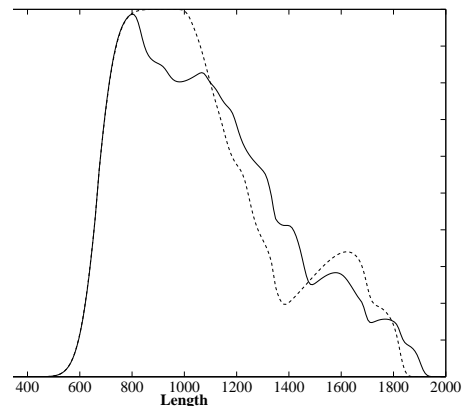

(a)

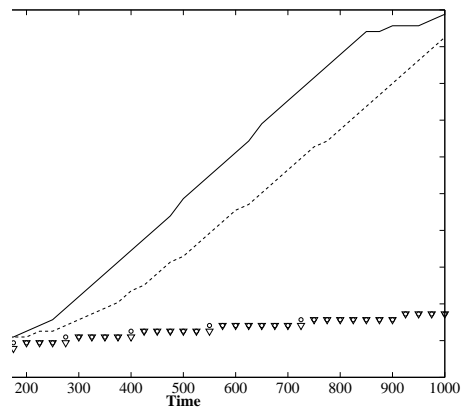

(b)

Figure 3: Effect of Korteweg stress on forward fingering $R=2$, rear interface being stable (a) Band broadening curves for $P e=1000$ at $t=750$. The line represents the profile without Korteweg stress $(\delta=0)$ and the dashed line with $\delta=-10000$

(b) Mixing length for two interfaces in the presence and absence of Korteweg stress for $P e=1000$. The dashed line depicts the mixing length of unstable interface for $\delta=-10000$, the circle represents the mixing length of stable interface for $\delta=-10000$. The full line represents the mixing length for the unstable interface with $\delta=0$ and the triangle the stable interface mixing length for $\delta=0$ 


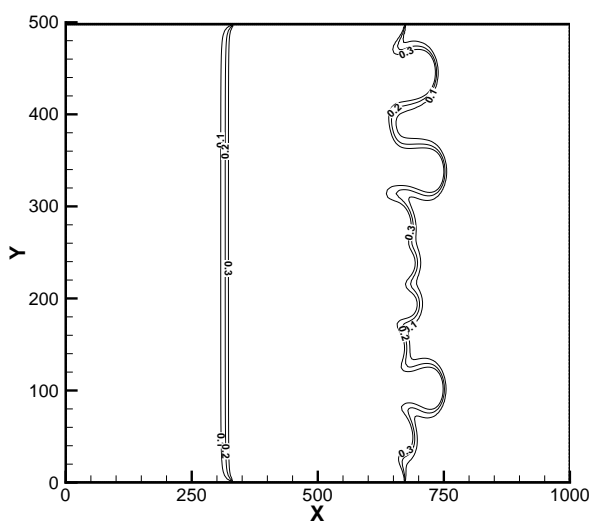

(a)

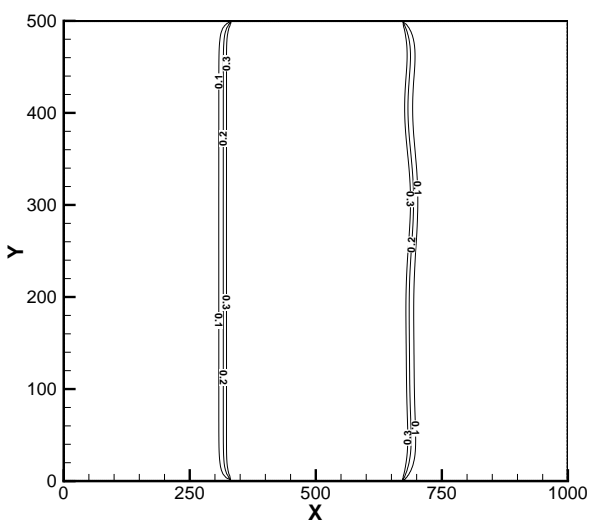

(b)

Figure 4: Concentration contours for forward fingering in the presence and absence of Korteweg stress for $P e=500, t=200, R=2, A=2$
(a) $\delta=0$
(b) $\delta=-10000$ 


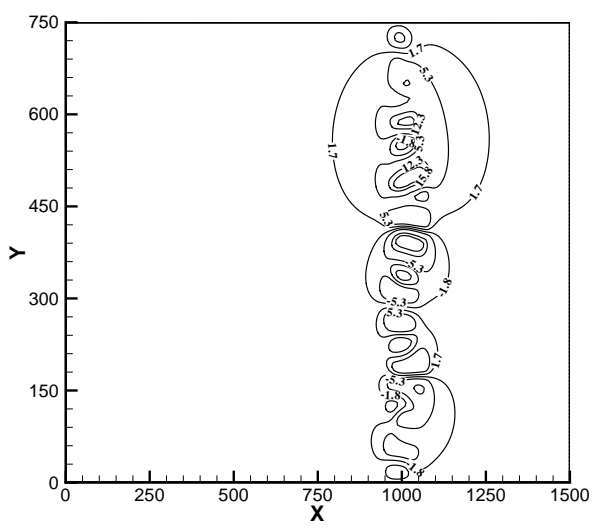

(a)

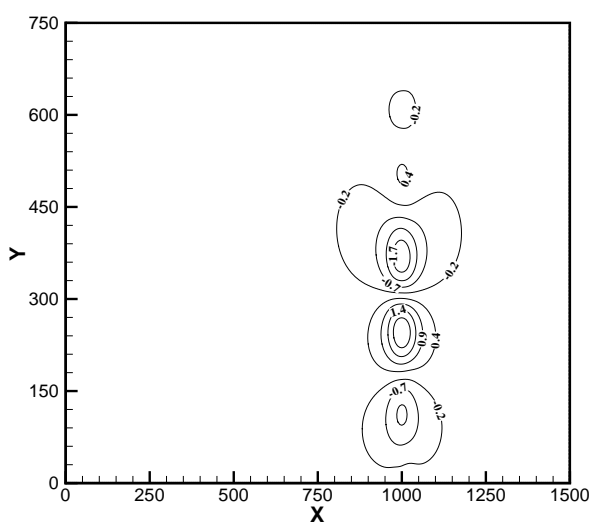

(b)

Figure 5: Stream function contours for forward fingering for $P e=750, t=200, R=2$, $A=2$
(a) $\delta=0$
(b) $\delta=-10000$ 
velocity and so the Korteweg stresses result in a stabilization and a lower magnitude of velocity.

To examine the stabilizing influence of the Korteweg stresses we have carried out a simulation where the Korteweg stresses are absent for an initial time period (until $t=50$ ). The parameters chosen for this were $P e=1000$, $R=2, A=2$. We see that the front interface exhibits a deformation and a fingering instability at $t=200$ when $\delta=0$ for the entire time period, Fig. 6(a). After a time instant $t=50$ the effect of Korteweg stresses were switched on by setting the parameter $\delta=-10000$. We found that the Korteweg stresses included at this later instant of time could stabilize the unstable front interface, Fig. 6(b). We have observed a critical time above which the Korteweg stresses could not stabilize the front face. This is to be expected since after a sufficiently long time the concentration gradients are reduced significantly by diffusion and hence even if we activate $\delta$ the effect of the Korteweg stresses are not strong enough for stabilization to occur. The results of this analysis is depicted in Fig. 6(a) and 6(b). The former depicts the concentration contours at $t=200$ when there are no Korteweg stresses. We clearly see the fingering in the front interface. The latter shows the concentration contours again at $t=200$ when the Korteweg stresses are activated at $t=50$. We can see the stabilization of the interface due to the Korteweg stresses.

Figure 7 displays the effect of $P e$ on the flow behavior of the system. In view of our scaling the diffusion coefficient also occurs in the Korteweg stress parameter $\delta$. So a change in $D$ would also imply a change in $\delta$. However since $\delta$ is kept fixed in the figures the effect of changing $P e$ is captured only in the length of the system. Note however that for these results the initial length of the slug is kept at one-third of the length of the corresponding system, centered around the middle line. Fig. 7(a) depicts the band broadening observed for two different $P e$. At $t=1000$ we see that for the higher $P e$ of 1000 the system exhibits relatively longer fingers compared to the lower $P e$ of 500. However for $P e=500$ the fingers have almost reached the right end of the system. We have used the homogeneous Dirichlet boundary condition $c=0$ at the right end of the system and this explains the sharp concentration gradient at $A P e=1000$. We note here that for the higher $P e$ the right boundary is further away from the slug as compared to the lower Pe. Hence the effect of the right boundary is less prominent for the higher $P e$. To understand the evolution of the system with time we depict in Fig. 7(b) the mixing lengths corresponding to the two interfaces for the two different values 


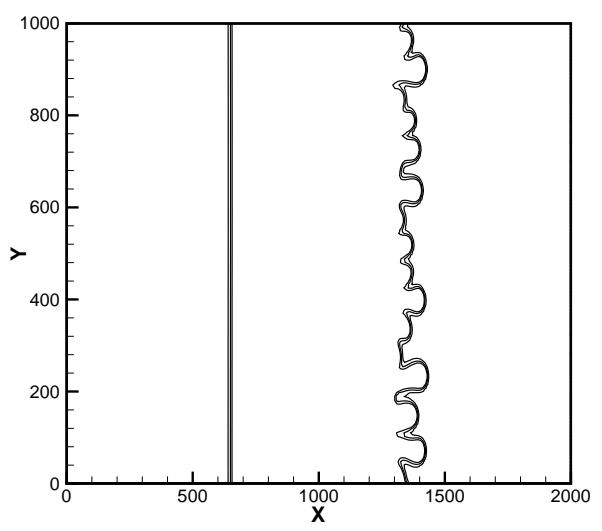

(a)

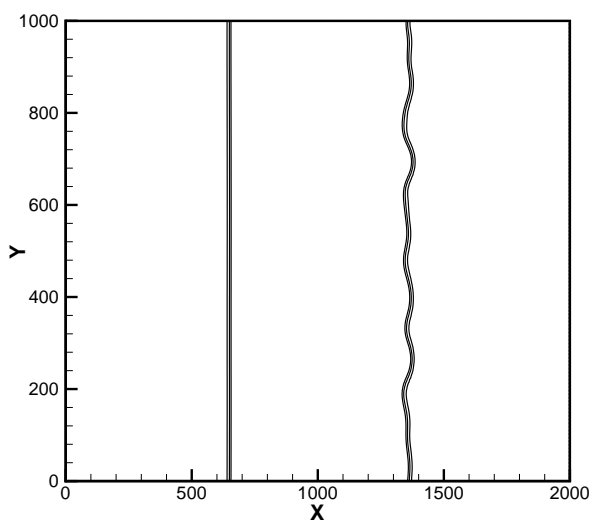

(b)

Figure 6: Concentration contours for forward fingering for $P e=1000, t=200, R=2$, $A=2$
(a) $\delta=0$
(b) $\delta=0$ till $t=50$ and then $\delta=-10000$ 


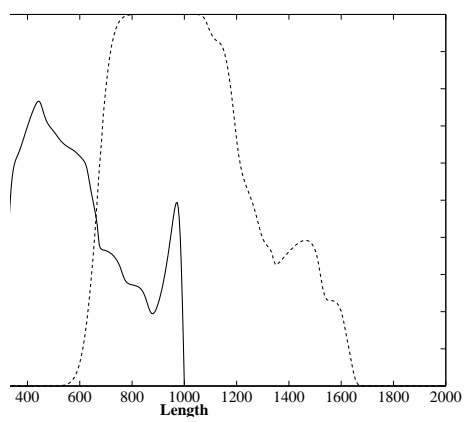

(a)

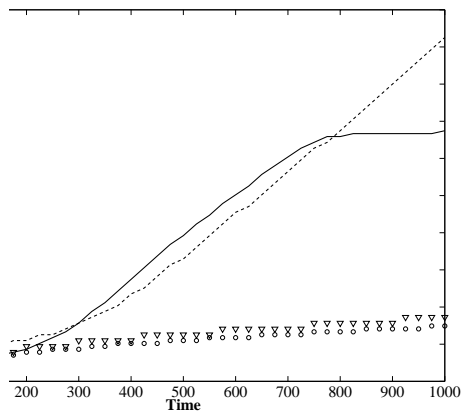

(b)

Figure 7: Effect of $P e$ for forward fingering $R=2, \delta=-10000$, rear interface being stable (a) Band broadening at $t=1000$. The full line depicts the transverse average concentration profiles for $P e=500$ and the dashed line shows the same for $P e=1000$

(b) Mixing length for two interfaces. The full line depicts the unstable interface and the circle represents the stable interface for $P e=500$. The dashed line shows the unstable interface and the triangle displays the situation for the stable interface for $P e=1000$ 
of $P e$. This figure clearly indicates that mixing length is a valuable asset in evaluating qualitative behavior: in the early stages of the evolution say for $t<700$ we see that the two unstable mixing lengths evolve quantitatively in a similar manner. We attribute the small differences in the mixing lengths in these periods to the variations in the random initial perturbation. We see that the mixing lengths for the lower $P e$ show a saturation since the fingers have reached the end of the system. For these larger values of time the two mixing length curves diverge from each other and the mixing length for the higher $P e$ case is more than that of the lower $P e$ case. This is consistent with the profiles of the transversely averaged concentrations shown in Fig. 7(a). For the stable interface also we see that the mixing length curves corresponding to the two $P e$ are quantitatively identical. The scaling for both the values of $P e$ is as $t^{1 / 2}$. So we can conclude from the mixing lengths that changing the length and height of the sample has no influence on the fingering behavior.

So far we have focused on the parameter $R>0$. We would like to now compare the behavior of the system when $R<0$ with $R>0$. In Fig. 8(a) we depict the behavior of the system when we have no Korteweg stresses. For $R>0$ the front face is unstable and the rear face is stable. For $R<0$ the reverse holds. The mixing length for both faces scales in the same manner when the interface is stable. This is true since the dependency on time is now decided solely by diffusion which is equal for both cases. There is a difference between the mixing lengths of the unstable interfaces. Specifically we find that the unstable backward mixing does not progress as fast as the forward fingering for larger time values when Korteweg stresses are absent. The mixing lengths for the forward fingering are hence higher than that of the backward fingering after the effect of the initial perturbations have decayed. Figure 8(b) shows the behavior when the effect of Korteweg stresses are included. We again see that the respective stable interfaces scale as $t^{1 / 2}$. The mixing lengths for the corresponding unstable interfaces has now decreased. For instance the forward mixing length when $\delta=0$ is around 490 compared to the case when $\delta=-10000$ where the mixing length is 460. A similar decrease in the mixing length is observed for the backward face when the effect of $\delta$ is included. This is attributed to the stabilizing influence of the Korteweg stresses. Here we would like to point out that similar differences in the backward and forward mixing lengths have been observed in the past (Mishra et al. (2008)). 


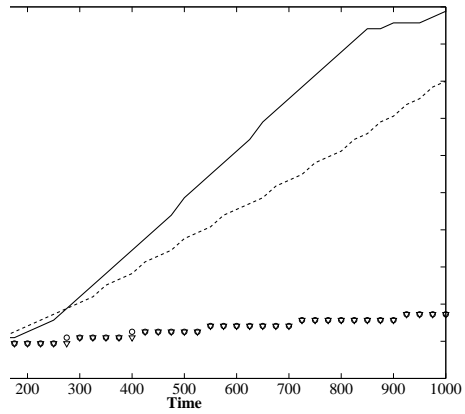

(a)

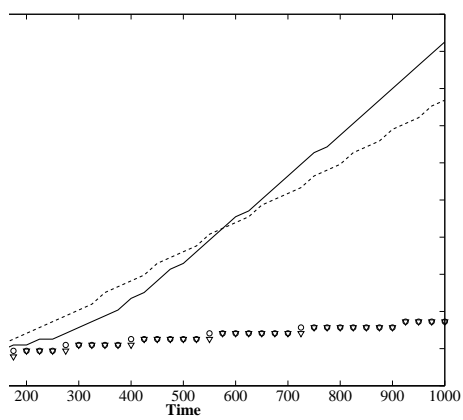

(b)

Figure 8: Comparison of the mixing lengths for forward $(R=2)$ and backward $(R=-2)$ fingering profiles for $P e=1000$

(a) $\delta=0$. The full line and circles show the unstable and stable interfaces respectively for $R=2$. The dashed line and triangles display the unstable and stable interfaces for $R=-2$

(b) $\delta=-10000$. The full line and circles shows the unstable and stable interfaces respectively for $R=2$. The dashed line and triangles displays the unstable and stable interfaces for $R=-2$ 


\section{Conclusion}

In this paper we have first shown that it is possible to have a unified approach to tackle both miscible as well as immiscible fingering when we include the Korteweg stresses for the former and the continuum theory of surface tension for the latter. This approach has also allowed us to identify the sign of the constants which arise in the Korteweg stresses from a dynamic or kinetic perspective.

We have further analyzed the influence of Korteweg stresses on the behavior of this system and found that they have a stabilizing influence. This is not surprising as surface tension has a stabilizing influence on immiscible fingering. Similarly diffusivity has a stabilizing influence on miscible fingering as it serves to reduce the concentration gradients and hence the viscosity gradients which are responsible for inducing the fingering. An important point to note here is that the mixing length curves as well as the band broadening curves are generated by following the time evolution of random perturbations given to the concentration at the slug interface and hence are stochastic in nature. This must be kept in mind when comparing the mixing length curves at initial stages of evolution when the effect of the initial perturbation is likely to be present.

One of the primary motivations of this work is to provide a platform for understanding Korteweg stresses in a comprehensive manner. This will help us understand how Korteweg stresses can be controlled. For instance it is a well known fact that surface tension can be controlled by adding surfactants. Similarly if we can determine methods by which Korteweg stresses can be affected we could think in terms of using this as a control option of our system. This would then enable us to have a dominant role for Korteweg stresses even when gravity effects are important.

\section{References}

Y. Nagatsu, A. Hayashi, M. Ban, Y. Kato, Y. Tada, Spiral pattern in a radial displacement involving a reaction-producing gel, Phys. Rev. E 78 (2008) 026307(1-6).

C.-S. Yih, Stratified Flows, Academic Press, second edn., 1980.

G. M. Homsy, Viscous Fingering in Porous media, Ann. Rev. Fluid. Mech 19 (1987) 271-311. 
R. A. Shalliker, H. J. Catchpoole, G. R. Dennis, G. Guiochon, Visualising viscous fingering in chromatography columns: High viscosity solute plug, J. Chromatography A 1142 (2007a) 48-57.

R. A. Shalliker, V. Wong, G. Guiochon, Reproducibility of the finger pattern in viscous fingering, J. Chromatography A 1161 (2007b) 121-131.

C. T. Tan, G. M. Homsy, Simulation of non-linear viscous fingering in miscible displacement, Phys. Fluids 31 (1988) 1330.

W. B. Zimmerman, G. M. Homsy, Nonlinear viscous fingering in miscible displacement with anisotropic dispersion, Phys. Fluids A 3 (1991) 1859.

C.-Y. Chen, L. Wang, E. Meiburg, Miscible droplets in a porous medium and the effects of Korteweg stresses, Phy. Fluids 13 (2001) 2447-2456.

D. Joseph, A. Huang, H. Hu, Non-solenoidal velocity effects and Korteweg stresses in simple mixture of incompressible liquids, Physica D 97 (1996) 104.

N. Bessonov, V. A. Volpert, J. A. Pojman, B. D. Zoltowski, Numerical Simulations of Convection Induced by Korteweg Stresses in Miscible PolymerMonomer Systems, Microgravity Sci. Tech 17 (2005) 2-6.

J. U. Brackbill, D. B. Kothe, C. Zemach, A continuum method for modeling surface tension, J. Chromatography A 100 (1992) 335-354.

X. Guan, R. Pitchumani, Viscous Fingering in a Hele-Shaw Cell With Finite Viscosity Ratio and Interfacial Tension, J. Fluids Eng 125 (2003) 354-364.

B. T. Nadiga, S. Zaleski, Investigations of a Two-phase Fluid Model, Eur. J. Mech. B/Fluids 15 (1996) 885.

Q. Zou, X. He, Derivation of the macroscopic continuum equations for multi phase flow, Phys. Rev E 59 (1999) 1253-1255.

C. T. Tan, G. M. Homsy, Stability of miscible displacements in porous media: Rectilinear flow, Phys. Fluids 29 (1986) 3549-3556.

G. Rousseaux, A. De Wit, M. Martin, Viscous fingering in packed chromatographic columns: linear stability analysis, J. Chromatography A 1149 (2007) 254-273. 
M. Mishra, M. Martin, A. De Wit, Miscible viscous fingering with linear adsorption on the porous matrix, Phys. Fluids 19 (2007) 073101-(1-9).

M. Mishra, M. Martin, A.De Wit, Differences in miscible viscous fingering of finite width slices with positive or negative log mobility ratio, Phys. Rev E 78 (2008) 066306-(1-11).

C.-Y. Chen, S.-W. Wang, Miscible displacement of a layer with finite width in porous media, Int. Journal of numerical methods for heat and fluid flow 11 (8) (2001) 761-778.

H. Hu, D. D. Joseph, Miscible displacement in a Hele-Shaw cell, Z. Angew. Math. Phys 43 (1992) 626.

S. Swernath, S. Pushpavanam, Viscous fingering in a horizontal flow through a porous medium induced by chemical reactions under isothermal and adiabatic conditions, J. Chem. Phys 127 (2007) 204701(1-14). 